\title{
Fortbildung für Ärzte und Apotheker „Phytopharmaka und Phytotherapie“ der Gesellschaft für Phytotherapie (GPT) startet erneut 2017
}

Die Phytotherapie ist heute ein unverzichtbarer Teil der Therapie in der modernen Medizin. Für Ärzte und Apotheker ist es daher wichtig, die Pflanzen, ihre Wirkstoffe und deren Kombinationen hinsichtlich der vielen verschiedenen Erkrankungen und Indikationen besonders gut zu kennen. Eine Fortbildung ist dazu dringend notwendig.

Die Gesellschaft für Phytotherapie (GPT) bietet deshalb 2017 und 2018 vier Fortbildungsmodule für Ärzte und Apotheker an, in denen namhafte Referenten in 3-tägigen Seminaren nach Indikationsgebieten geordnet eine Vielzahl von Heilpflanzen mit ihren Inhaltsstoffen und deren Wirkung auf den menschlichen Organismus vorstellen sowie konkrete Fallbeispiele besprechen.

Ziel ist es, neue Erkenntnisse einschließlich klinischer Studien zu Phytopharmaka und ihrer Anwendung in der ärztlichen Praxis und in der Apotheke zu vermitteln. Die Teilnehmer werden erfahren, dass die Wissenschaft vor der Phytotherapie nicht Halt gemacht hat und mittlerweile die Wirkungen vieler pflanzlicher Arzneimittel gut dokumentiert sind.

\section{Module}

Es sind wieder 4 Module geplant, die im Frühjahr und Herbst 2017 und 2018 stattfinden werden und mit einem Zertifikat enden. Das 1. Modul findet vom 31.3.-2.4.2017 im Lindner Sport \& Aktivhotel Kranichhöhe in 53804 Much/Bergi-

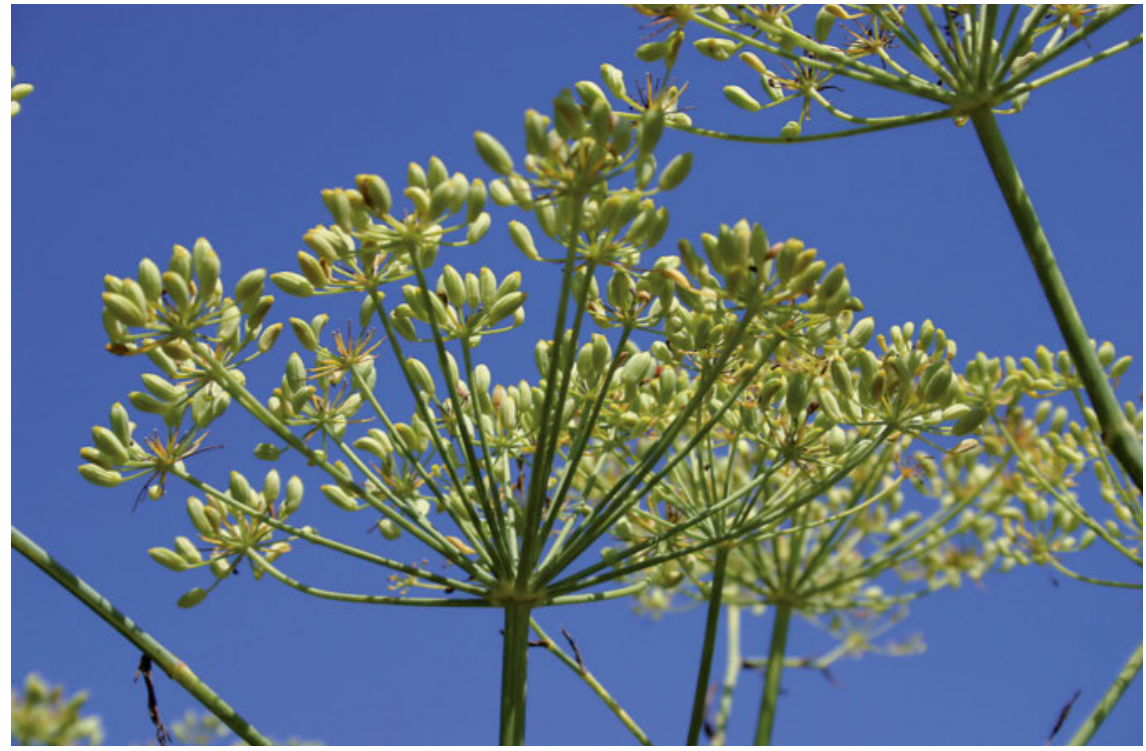

Von der Pflanze zur Indikation mit Fallbeispiel aus der Praxis - das sind Inhalte der GPT-Fortbildungsreihe. (c) Michael Zimmermann/Thieme Verlagsgruppe

sches Land statt. Die Themen des 1. Moduls sind:

- Allgemeine Einführung in die Phytotherapie

- Phytotherapie in der Psychiatrie

- Phytotherapie bei Schlafstörungen

- Phytotherapie bei Hauterkrankungen

- Phytotherapie bei rheumatischen Erkrankungen

- Abgrenzung Phytopharmaka/Nahrungsergänzungsmittel.

Der konkrete Ablaufplan des 1. Moduls ist zu finden unter: http://phytotherapy.org/ de/termine/fortbildungsveranstaltungen/

Der Unkostenbeitrag beträgt 390,Euro einschließlich Kursgebühren, Kursmaterialien und Verpflegung. Das Hotel hat ein Kontingent zum Sonderpreis von $103,-€$ pro Nacht bis 8 Wochen vor Veranstaltungsbeginn hinterlegt. Das Modul wird von der Landesärzte- und Landesapothekerkammer mit Fortbildungspunkten akkreditiert.

Erfahren Sie mehr über Nutzen, Interaktionen und Nebenwirkungen von Phytopharmaka als Bestandteil einer modernen Medizin und melden Sie sich an unter:

\section{Gesellschaft für Phytotherapie e.V. \\ Cornelia Schwöppe, Schatzmeisterin \\ Hebborner Berg 51 \\ 51467 Bergisch Gladbach \\ schwoeppe@phythotherapie.de \\ Tel.: 0172/5710117 \\ Fax: 02202/9790363}

